\title{
Most Cited Articles in 2019
}

Dear Readers

We would like to announce the top 11 articles most frequently cited in the 2017 and 2018 issues of the International Heart Journal. The numbers of citations are cited in the year of 2019.

\begin{tabular}{|c|c|c|c|}
\hline Rank & Authors/Title/Issue & Type & $\begin{array}{l}\text { Number } \\
\text { of citation }\end{array}$ \\
\hline 1 & $\begin{array}{l}\text { Haruki Imamura, Takashi Yamaguchi, Daiji Nagayama, Atsuhito Saiki, Kohji } \\
\text { Shirai, Ichiro Tatsuno. Resveratrol Ameliorates Arterial Stiffness Assessed by } \\
\text { Cardio-Ankle Vascular Index in Patients With Type } 2 \text { Diabetes Mellitus. 2017; } \\
\text { 58(4): 577-583. }\end{array}$ & Clinical Study & 16 \\
\hline 2 & $\begin{array}{l}\text { Yuichi Sasaki, Yoshiyuki Ikeda, Masaaki Iwabayashi, Yuichi Akasaki, Mitsuru } \\
\text { Ohishi. The Impact of Autophagy on Cardiovascular Senescence and Diseases. } \\
\text { 2017; 58(5): 666-673. }\end{array}$ & Review & 15 \\
\hline 3 & $\begin{array}{l}\text { Ryo Naito, Katsumi Miyauchi. Coronary Artery Disease and Type } 2 \text { Diabetes } \\
\text { Mellitus: Current Treatment Strategies and Future Perspective. 2017; 58(4): 475- } \\
480 .\end{array}$ & Review & 14 \\
\hline 4 & $\begin{array}{l}\text { Satoshi Kodera, Arihiro Kiyosue, Jiro Ando, Hiroshi Akazawa, Hiroyuki Morita, } \\
\text { Masafumi Watanabe, Issei Komuro. Cost-Effectiveness Analysis of Cardiovascular } \\
\text { Disease Treatment in Japan. 2017; 58(6): 847-852. }\end{array}$ & Review & 9 \\
\hline 4 & $\begin{array}{l}\text { Shouwen Zhang, Rongjun Zhang, Fangfang Wu, Xinhua Li. MicroRNA-208a } \\
\text { Regulates H9c2 Cells Simulated Ischemia-Reperfusion Myocardial Injury via } \\
\text { Targeting CHD9 through Notch/NF-kappa B Signal Pathways. 2018; 59(3): 580- } \\
588 .\end{array}$ & $\begin{array}{l}\text { Experimental } \\
\text { Study }\end{array}$ & 9 \\
\hline 6 & $\begin{array}{l}\text { Wentao Xiao, Suping Guo, Chuanyu Gao, Guoyou Dai, Yongjv Gao, Muwei Li, } \\
\text { Xianpei Wang, Dayi Hu. A Randomized Comparative Study on the Efficacy of } \\
\text { Intracoronary Infusion of Autologous Bone Marrow Mononuclear Cells and } \\
\text { Mesenchymal Stem Cells in Patients With Dilated Cardiomyopathy. 2017; 58(2): } \\
\text { 238-244. }\end{array}$ & Clinical Study & 8 \\
\hline 6 & $\begin{array}{l}\text { Kyung-Kuk Hwang, Sang-Yong Eom, Sang Yeub Lee, Sang Min Kim, Myeong- } \\
\text { Chan Cho, Young Jo Kim, Ki Bae Seung, Myung Ho Jeong, Jang-Whan Bae, Other } \\
\text { Korea Acute Myocardial Infarction Registry Investigators. Atrial Fibrillation on } \\
\text { Admission Is Related With Higher Mortality in ST-Segment Elevation Myocardial } \\
\text { Infarction Patients: Lessons From the Korea Acute Myocardial Infarction Registry } \\
\text { (KAMIR). 2017; 58(4): 486-494. }\end{array}$ & Clinical Study & 8 \\
\hline 6 & $\begin{array}{l}\text { Naoko Matsushita, Nanae Ishida, Miho Ibi, Maki Saito, Atsushi Sanbe, Hisashi } \\
\text { Shimojo, Satoshi Suzuki, Hermann Koepsell, Yasuchika Takeishi, Yoshihiro } \\
\text { Morino, Eiichi Taira, Yohei Sawa, Masamichi Hirose. Chronic Pressure Overload } \\
\text { Induces Cardiac Hypertrophy and Fibrosis via Increases in SGLT1 and IL-18 Gene } \\
\text { Expression in Mice. 2018; 59(5): 1123-1133. }\end{array}$ & $\begin{array}{l}\text { Experimental } \\
\text { Study }\end{array}$ & 8 \\
\hline 6 & $\begin{array}{l}\text { Dae-Won Kim, Sung-Ho Her, Mahn-Won Park, Jung Sun Cho, Tae-Seok Kim, } \\
\text { Hyeonjeong Kang, Doo Sun Sim, Young Joon Hong, Ju Han Kim, Youngkeun Ahn, } \\
\text { Kiyuk Chang, Wook-Sung Chung, Ki-Bae Seung, Myung-Ho Jeong, Tai-Ho Rho. } \\
\text { Impact of Postprocedural TIMI Flow on Long-Term Clinical Outcomes in Patients } \\
\text { with Acute Myocardial Infarction: Five Year Follow-Up Results in the Corea-AMI } \\
\text { Registry. 2017; 58(5): 674-685. }\end{array}$ & Clinical Study & 8 \\
\hline 6 & $\begin{array}{l}\text { Xiaochun Lu, Dawei Yin, Bo Zhou, Tieling Li. MiR-135a Promotes Inflammatory } \\
\text { Responses of Vascular Smooth Muscle Cells From db/db Mice via Downregulation } \\
\text { of FOXO1. 2018; 59(1): 170-179. }\end{array}$ & $\begin{array}{l}\text { Experimental } \\
\text { Study }\end{array}$ & 8 \\
\hline 6 & $\begin{array}{l}\text { Yajing Mao, Jun-ichiro Koga, Masaki Tokutome, Tetsuya Matoba, Gentaro Ikeda, } \\
\text { Kaku Nakano, Kensuke Egashira. Nanoparticle-Mediated Delivery of Pitavastatin } \\
\text { to Monocytes/Macrophages Inhibits Left Ventricular Remodeling After Acute } \\
\text { Myocardial Infarction by Inhibiting Monocyte-Mediated Inflammation. 2017; } \\
\text { 58(4): 615-623. }\end{array}$ & $\begin{array}{l}\text { Experimental } \\
\text { Study }\end{array}$ & 8 \\
\hline
\end{tabular}

We appreciate all authors who submitted their manuscripts to the International Heart Journal, and look forward to receiving manuscripts with high scientific impact from all over the world.

Issei Komuro, MD

Editor-in-Chief

International Heart Journal

November 2020 